\title{
BIOLOGÍA Y COMPORTAMIENTO SEXUAL DEL MUTANTE OJOS AMARILLOS DE ANASTREPHA LUDENS (DIPTERA: TEPHRITIDAE)
}

\author{
José Luis Quintero Fong1, José Salvador Meza Hernández1, \\ Dina OROZCo DÁVILA ${ }^{1}$, Miguel Salvador FiguEROA ${ }^{2}$ y Leopoldo CRUZ-LóPEZ ${ }^{3}$ \\ ${ }^{1}$ Programa Moscamed Moscafrut-Desarrollo de Métodos. Central Poniente No. 14 altos-Esq. $2^{\text {a }}$. \\ Avenida Sur. CP 30700. Tapachula, Chiapas, MÉXICO. \\ quinterofong@hotmail.com \\ 2 Área de Biotecnología. Universidad Autónoma de Chiapas, Carretera a Puerto Madero, Km. 1.5, \\ Tapachula, Chiapas, MÉXICO. \\ ${ }^{3}$ El Colegio de la Frontera Sur, Apdo. Postal 36, Carretera Antiguo Aeropuerto, 30700, Tapachula, \\ Chiapas, MÉXICO.
}

Quintero Fong, J. L., J. S. Meza Hernández, D. Orozco Dávila, M. S. Figueroa y L. Cruz-López. 2009. Biología y comportamiento sexual del mutante ojos amarillos de Anastrepha ludens (Diptera: Tephritidae). Acta Zool. Mex. (n. s.) 25(1):9-20.

RESUMEN. Se comparó la biología y el comportamiento sexual entre una cepa de laboratorio con fenotipo silvestre (LFS) con una cepa de laboratorio con fenotipo mutante (LFM) de Anastrepha ludens (Loew), que se caracteriza por una coloración del cuerpo clara y ojos amarillo cremoso, para determinar su potencial como marcador genético en los programas de control mediante el uso de la técnica del insecto estéril. Los resultados del estudio indicaron que los individuos de la cepa mutante LFM no difieren significativamente de los individuos de la cepa LFS respecto a su fecundidad, fertilidad y supervivencia de inmaduros, así como en la emisión de feromona sexual. Esto es indicativo de que podría ser adaptada a las condiciones de cría masiva. Sin embargo, las pruebas de apareamiento indicaron que aunque los insectos LFM copularon con los insectos de campo de fenotipo silvestre (CFS), presentaron cierto grado de aislamiento y bajos porcentajes de apareamiento de los machos tanto con hembras CFS y LFS, lo cual implica que no sea recomendable el uso de la cepa mutante LFM como marcador genético en programas que utilizan la técnica del insecto estéril, para el control de la mosca Mexicana de la fruta.

Palabras clave: Anastrepha ludens, comportamiento sexual, cría masiva, mutante, marcador genético.

Quintero Fong, J. L., J. S. Meza Hernández, D. Orozco Dávila, M. S. Figueroa \& L. Cruz-López. 2009. Biology and sexual behavior of the yellow eyes mutant Anastrepha ludens (Diptera: Tephritidae). Acta Zool. Mex. 25(1):37-48.

ABSTRACT. We compared the biology and sexual behavior between a strain of laboratory with wild phenotype (LFS) and a strain of laboratory with mutant phenotype (LFM) of Anastrepha ludens (Loew), characterized by clear coloration of the body and creamy yellow eyes to determine their potential as genetic marker in the control programmes through the use of the sterile insect technique. The survey results indicated that individuals of the LFM mutant strain not differ significantly from individuals of the LFS strain respect to their fecundity, fertility and survival of immature stages, as 
well as in the emission of sexual pheromone. This indicates that could be adapted to mass-rearing. However, mating tests indicated that although the LFM insects mated with the insects of field of wild phenotype (CFS), presented some degree of isolation and low percentages of mating of males with both LFS and CFS females, implying that is not recommended the use of the LFM mutant strain as genetic marker in programs that use the sterile insect technique, for the control of the Mexican fruit fly.

Keys Words: Anastrepha ludens, sexual behavior, mass-rearing, mutant, genetic marker.

\section{INTRODUCCIÓN}

Anastrepha ludens (Loew 1873), conocida como mosca Mexicana de la fruta, es una plaga importante en cultivos de cítricos y mango (Norrbom \& Kim 1988). Esta especie ha sido registrada desde el sur de Texas hasta Costa Rica (Hernández-Ortíz \& Aluja 1993, Norrbom et al. 2000).

Por el daño económico que $A$. ludens ocasiona en frutos de huertos comerciales, se han desarrollado diversos métodos de control. En la actualidad su control se realiza a través de programas de manejo integrado de plagas (MIP), que son aplicados a nivel regional y nacional para el establecimiento de áreas libres de plagas (Reyes et al. 2000, Enkerlin 2005). Los métodos de control más comunes usados en estos programas han sido la aplicación de cebos tóxicos (por ejemplo, malatión-proteína hidrolizada) y la Técnica del Insecto Estéril (TIE). Esta última, por ser ambientalmente amigable, ha sido usada con éxito por muchos programas de control de plagas de importancia agrícola (Knipling 1953, Parker \& Mehta 2007). Para estimar el nivel de eficiencia de la TIE, en los programas de control, es necesario contar con un procedimiento que permita discriminar, inequívocamente, a los insectos liberados de los silvestres (Enkerlin et al. 1996). En este sentido el método de Norris (1957) ha sido usado con modificaciones durante muchos años por todas las plantas de cría masiva de moscas de la fruta del mundo. Para el caso de $A$. ludens, las pupas son marcadas externamente, con una mezcla de una sustancia fluorescente y polvo seco (Day-Glo fluorescent, neon red, Cleveland, Ohio) interiorizada por el insecto durante el proceso de emergencia del adulto (Schroeder \& Mitchell 1981). A pesar de que el marcaje fluorescente de moscas ha tenido éxito, éste presenta algunos problemas que van desde su costo (alto), hasta su efecto sobre la calidad de los insectos (Coppedge et al. 1979). Además, requiere trabajo intensivo y con efectos negativos para la salud de los trabajadores que realizan el pintado de las pupas dentro de las plantas productoras (Khoury \& Abi 2001). Por lo tanto se necesita mejorar el proceso o desarrollar técnicas alternas para el marcaje de moscas producidas masivamente (Hagler \& Jackson 2001).

Aunque recientemente se han reportado diversos métodos de marcaje de insectos, algunos no pueden ser aplicados en el campo o requieren de mucho tiempo para el análisis de diferenciación (Hangler \& Jackson 2001). Saul y McCombs (1992) trabajaron con marcadores de ojos en tres especies de moscas de la fruta; Niyazi et al. (2005) emplearon la mutante $S r^{2}$ en la mosca del Mediterráneo Ceratitis capitata 
(Wiedemann); mientras que Yamada y Selivon (2001) estudiaron el mutante de coloración de ojos "Rose" (ro). Todos estos trabajos proponen el empleo de moscas marcadas genéticamente como sustituto de la tinción con polvo fluorescente. Aunque esta alternativa pudiera reducir el costo del proceso, mejorar la calidad de los insectos y facilitar la identificación positiva, en los trabajos con marcadores de color de ojos, no se incluyeron los estudios sobre el comportamiento sexual, esenciales para una completa recomendación del uso de estas mutantes en la TIE.

Meza et al. (2004) documentaron una mutante espontánea en la cría masiva de $A$. ludens. Además de describir que el mutante tiene ojos amarillos, cuerpo claro y que es un locus trasmitido como recesivo y autosomal, dichos autores sugirieron que ese mutante tiene potencial para emplearse como un marcador en la liberación masiva de insectos estériles o en estudios de liberación y recaptura.

La falta de información respecto al comportamiento sexual de mutantes de color de ojo, imposibilita la evaluación certera del potencial que esta alternativa tiene para su uso en la TIE. El objetivo del presente trabajo fue estudiar los atributos biológicos y el comportamiento sexual de la mutante de Anastrepha ludens de ojos amarillos y cuerpo claro, con el fin de discutir su posible uso como marcador genético en programas de liberación de la TIE.

\section{MATERIAL Y MÉTODOS}

Material biológico. En este trabajo se emplearon adultos de A. ludens con fenotipo silvestre (obtenidos en laboratorio y en campo) y con fenotipo mutante (obtenidos en laboratorio). Los insectos adultos de laboratorio con fenotipo silvestre (LFS), fueron obtenidos a partir de pupas producto de la cría masiva de $A$. ludens, en la Planta Moscafrut de Metapa de Domínguez, Chiapas, México. Los insectos adultos de campo con fenotipo silvestre (CFS), se obtuvieron mediante la recuperación de larvas de naranja agria (Citrus aurantium, L.) colectadas en la región del Soconusco, Chiapas, México. Los insectos adultos de laboratorio con fenotipo mutante (LFM) (Meza et al. 2004), se obtuvieron como pupas de una colonia que ha sido mantenida en el laboratorio de evaluaciones de campo de la Planta Moscafrut de Metapa de Domínguez, Chiapas, México. Esta cepa aislada de una pareja de individuos, para evitar la reducción del acervo genético y su posible disminución de sus atributos biológicos, fue cruzada con insectos silvestres, para restaurar su competitividad de acuerdo a lo documentado por Rull y Barreda-Landa (2007). La cruza fue realizada con mil hembras mutantes y mil machos silvestres. Debido a que esta mutación es recesiva y autosomal (Meza et al. 2004), fue necesario realizar la cruza entre los híbridos de la progenie $\mathrm{F}_{1}$ para obtener la mutación de los individuos en la progenie $\mathrm{F}_{2}$. Este procedimiento fue similar al realizado por Harris et al. (2006), en el estudio de una mutación de las mosca del melón Bactrocera cucurbitae. Está restauración se realizó cinco veces antes de las evaluaciones. 
En la Figura 1 se muestra las diferencias fenotípicas entre los individuos LFS y LFM.

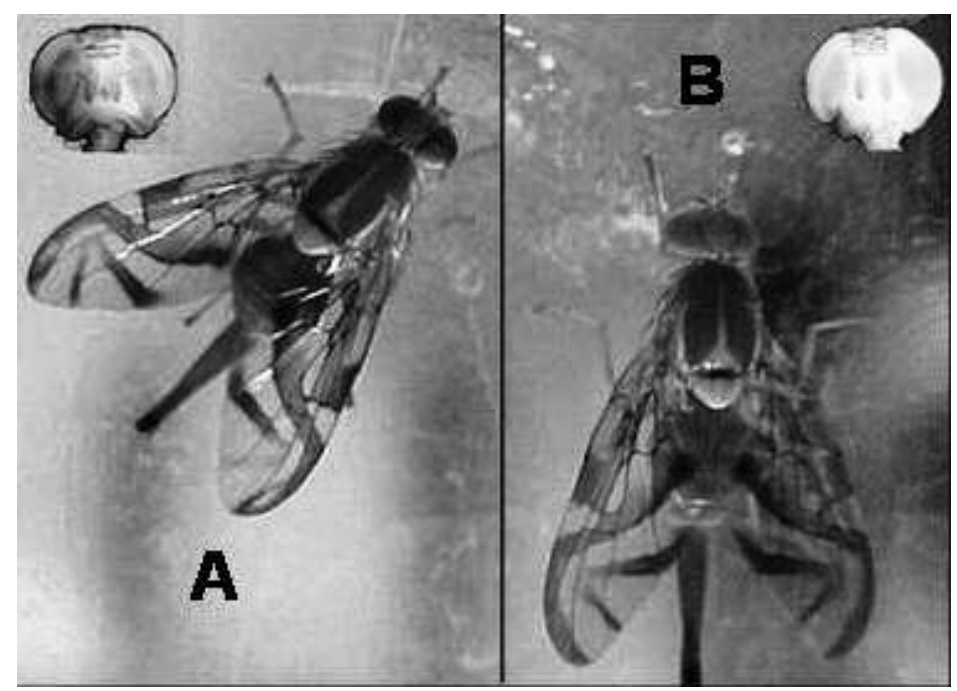

Figura 1. Hembras de A. ludens LFS (A) y LFM (B).

Manejo y condiciones de cría. En todos los experimentos se utilizaron adultos fértiles sexualmente maduros de 16 días de edad para los de campo (CFS) y de 10 días para los de laboratorio (LFS y LFM), cultivados de acuerdo a los procedimientos de manejo y cría empleados en la planta Moscafrut. (Hernández et al. 2005). Los adultos se alimentaron con una dieta formulada con agua y azúcar: proteína hidrolizada (proporción 3:1). En todas las pruebas las hembras y los machos fueron aislados al emerger en jaulas independientes (para evitar cualquier contacto de feromonas antes de la prueba) y mantenidos bajo un fotoperiodo de 12 horas luz (de

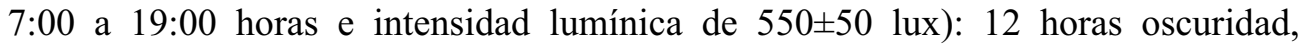
temperatura de $25 \pm 1 \infty \mathrm{C}$ y humedad relativa de $65 \pm 5 \%$.

Determinación de la fecundidad y fertilidad. Para determinar el grado de fertilidad y fecundidad tanto de los insectos LFS como los LFM, 40 parejas sexualmente maduras (diez días de edad), se colocaron en una jaula de plexiglass $(20 \times 20 \times 20 \mathrm{~cm})$ en condiciones ambientales similares a las descritas en el aislamiento de los insectos. Para la colecta de los huevecillos en la jaula se dispuso de un "panel de oviposición" (recipiente de forma cilíndrica de $7.5 \mathrm{~cm}$ de altura y $7.5 \mathrm{~cm}$ de diámetro, cubierta en la base con tela tergal y revestida con silicón en la parte interna), colocado en la parte superior de la jaula y humedecido con agua destilada. Los huevecillos se colectaron diariamente durante un periodo de 10 días. Después de su cuantificación, los huevecillos se colocaron en cámaras húmedas (cajas Petri de 
plástico, provistas de esponjas con agua) y se incubaron a $25^{\circ} \mathrm{C}$, hasta la emergencia de la larva. La cantidad de huevecillos ovipositados fue factor de medida de la fecundidad y el porcentaje de eclosión se empleó como una medida de la fertilidad. El experimento se repitió diez veces simultáneamente para ambas cepas.

Determinación de la supervivencia de estados inmaduros. Para determinar la supervivencia para cada una de las etapas de desarrollo de los insectos LFS y LFM (huevo, larva, pupa y adulto) se colocaron 100 huevecillos en una caja de Petri de plástico, que contenía $50 \mathrm{~g}$ de dieta larvaria. Las larvas, pupas y adultos obtenidos durante el proceso de cría para cada caja fueron contabilizados. En Total se realizaron 10 repeticiones.

Prueba de compatibilidad sexual. Las pruebas de compatibilidad sexual se realizaron en la finca "El Refugio" (finca productora de Mango, variedad Ataulfo), localizada a $14^{\circ} 55^{\prime}$ " 08.9 " N, 92 $16^{\prime} 34.2$ " W y 137 msnm, en Tapachula, Chiapas, México, mientras que las condiciones ambientales (temperatura, humedad relativa y luz) durante la prueba fluctuaron entre $29-25^{\circ} \mathrm{C}, 65-75 \%$, H.R. y 2340 a 160 lux.

En las jaulas de campo, la preparación del material y el marcaje de los insectos, se realizó siguiendo los métodos utilizados por Meza-Hernández y Díaz-Fleischer (2006). En una jaula se liberaron 20 parejas de moscas LFM y 20 parejas CFS. En otra jaula, como control, se liberaron 20 parejas de moscas LFS y 20 parejas CFS. En cada jaula se registró el número de cópulas para cada una de las cuatro posibles combinaciones y, con ellos, se calculó la proporción de apareamiento (PM siglas en ingles), el índice de aislamiento (ISI siglas en ingles) propuestos por Cayol et al. (1999) y el índice de esterilidad (RSI siglas en ingles) propuesto por McInnis et al. (1996), que en este caso sirvió para evaluar la elección de la hembra silvestre. El registro de las cópulas se realizó de las 15:00 a las 19:00 horas, considerado como el horario de mayor actividad sexual para A. ludens (Aluja et al. 1983). En total se evaluaron nueve jaulas para cada tratamiento.

Determinación de la competencia sexual. Para estimar la competitividad sexual de los machos LFS y LFM, estos fueron evaluados en competencia por dos tipos de hembras CFS y LFS de manera separada. Adicionalmente, se prepararon jaulas con machos LFS y LFM (sin competencia) con hembras LFS. Las pruebas se realizaron, bajo condiciones de laboratorio, en jaulas de 10x10x10 cm (cubierta con malla tull de $1 \mathrm{~mm}$ ), registrándose el número de apareamientos y en el caso de la competencia el tipo de apareamiento. El horario de observación fue similar a las pruebas de compatibilidad sexual. Se realizaron cien repeticiones de cada prueba, en bloques de veinte jaulas durante cinco días.

Producción de feromonas. Para determinar la composición de los volátiles durante el llamado sexual, tanto de los machos de la colonia LFM como la de los machos LFS, se emplearon machos de diez días de post-emergencia. El análisis se realizó por cromatografía de gases acoplado a un espectrómetro de masas (CG-SM). 
La colecta y las condiciones del análisis siguieron el procedimiento reportado por Meza et al. (2002). En total se realizaron 10 colectas de volátiles para cada una de las colonias.

Análisis de datos. Para determinar similitudes y/o diferencias entre los individuos de la colonia LFM y LFS, los datos obtenidos de cada evaluación fueron analizados mediante una comparación de medias para datos pareados ( $\mathrm{t}$-student a $\alpha=0.05$ ). Los datos de fertilidad, supervivencia de estados inmaduros y proporción de apareamientos, fueron normalizados por medio de la transformación arcoseno $\sqrt{X}$ para su análisis (Zar 1999). Para comparar las cantidades de los volátiles liberados por moscas LFS y LFM se empleo la prueba de Mann-Whitney. En todos los análisis se utilizó el programa StatView para Windows (SAS Institute Inc. Copyright,, 19921998), versión 5.

\section{RESULTADOS}

Fecundidad, fertilidad y supervivencia de estados inmaduros. Los resultados indican que la fecundidad expresada en huevos por hembra por día $(\mathrm{t}=0.71, \mathrm{df}=18$, $\mathrm{P}>0.05)$, la fertilidad de los huevos $(\mathrm{t}=-1.924, \mathrm{df}=18, \mathrm{P}>0.05)$ y la supervivencia de los estados inmaduros (huevo-larva $\mathrm{t}=-0.74, \mathrm{df}=18, \mathrm{P}>0.05$; larva-pupa $\mathrm{t}=1.79$, $\mathrm{df}=18, \mathrm{P}>0.05$; pupa-adulto $\mathrm{t}=2.09, \mathrm{df}=18, \mathrm{P}>0.05)$, entre las cepas LFM y LFS no fueron estadísticamente significativos (Cuadro 1).

Cuadro 1. Tabla de atributos biológicos de la Cepa LFM y LFS de A. ludens.

\begin{tabular}{lccc}
\hline Parámetro & \multicolumn{2}{c}{ Cepa } \\
\hline & & $L F S$ & $p$ \\
\hline Fecundidad (h/h/d) & $68.04 \pm 8.261 \mathrm{a}$ & $60.78 \pm 5.940 \mathrm{a}$ & 0.48 \\
Fertilidad (\%) & $86.78 \pm 1.150 \mathrm{a}$ & $89.59 \pm 0.920 \mathrm{a}$ & 0.07 \\
Supervivencia huevo-larva (\%) & $64.60 \pm 2.548 \mathrm{a}$ & $62.00 \pm 2.404 \mathrm{a}$ & 0.46 \\
Supervivencia larva-pupa (\%) & $97.90 \pm 0.504 \mathrm{a}$ & $99.00 \pm 0.298 \mathrm{a}$ & 0.09 \\
Supervivencia pupa-adulto (\%) & $97.40 \pm 0.792 \mathrm{a}$ & $99.00 \pm 0.333 \mathrm{a}$ & 0.08 \\
\hline
\end{tabular}

Media \pm error estándar con distinta letra en una fila indican diferencias significativas $(\mathrm{p} \leq 0.05)$ $\mathrm{h} / \mathrm{h} / \mathrm{d}=$ huevo/hembra/día

Compatibilidad sexual. La proporción registrada de apareamientos en la jaula (media \pm error estándar) para la evaluación de los insectos LFS fue de $0.75 \pm 0.05$, mientras que para la jaula con insectos LFM fue de $0.73 \pm 0.02$, ambas resultaron mayores a lo establecido (0.02) por la AIEA (2003) para aceptar los datos como analizables. Además estos no presentaron diferencia significativa entre ellas. 
El Cuadro 2, compara los tipos de apareamiento posibles, entre las cepas evaluadas (LFS y LFM), y se observa que las frecuencias de cópula más altas fueron las homotípicas (apareamientos entre la misma cepa), sin presentar diferencias significativas entre las cepas. Tampoco se observaron diferencias significativas de las cópulas registradas entre las hembras LFS y LFM con machos silvestres. Mientras que en las cópulas heterotípicas (entre cepas diferentes) donde la hembra fue silvestre, las cópulas logradas por los machos LFM fueron significativamente menores a las de machos LFS $(\mathrm{t}=4.010, \mathrm{df}=16, \mathrm{P}<0.05)$.

Cuadro 2. Porcentaje por tipo de apareamiento de la cepa LFM y LFS frente a insectos CFS de $A$. ludens.

\begin{tabular}{|c|c|c|c|c|c|c|}
\hline \multirow[t]{2}{*}{$\begin{array}{l}\text { Cepa } \\
\text { evaluada } \\
\end{array}$} & \multicolumn{4}{|c|}{$\begin{array}{c}\text { Tipos de apareamiento } \\
\text { (macho-hembra) }\end{array}$} & \multicolumn{2}{|c|}{ Índices de compatibilidad } \\
\hline & $\mathrm{CC}$ & $\mathrm{CL}$ & $\overline{\mathrm{LL}}$ & $\mathrm{LC}$ & ISI & RSI \\
\hline LFS & $23.85 \pm 3.21 \mathrm{a}$ & $16.51 \pm 1.30 \mathrm{a}$ & $39.83 \pm 2.30 \mathrm{a}$ & $19.81 \pm 2.07 \mathrm{~b}$ & $0.27 \pm 0.04 \mathrm{~b}$ & $0.47 \pm 0.06 \mathrm{~b}$ \\
\hline LFM & $31.07 \pm 3.18 \mathrm{a}$ & $20.08 \pm 1.70 \mathrm{a}$ & $40.52 \pm 3.30 \mathrm{a}$ & $08.33 \pm 1.54 \mathrm{a}$ & $0.43 \pm 0.03 \mathrm{a}$ & $0.22 \pm 0.04 \mathrm{a}$ \\
\hline
\end{tabular}

Media \pm error estándar con distinta letra en una columna indican diferencias significativas $(\mathrm{p} \leq 0.05)$

$\mathrm{L}=$ Insecto de laboratorio

$\mathrm{C}=$ Insecto de campo

El índice ISI el cual compara el número de apareamientos homotípicos y heterotípicos indicó, que los insectos LFM fueron significativamente menos compatibles que los insectos LFS con los insectos silvestres $(\mathrm{t}=-3.30, \mathrm{df}=16$, $\mathrm{P}<0.05$ ). Por consiguiente, el RSI usado para verificar la preferencia en el apareamiento de la hembra silvestre, mostró que ambas cepas prefirieron aparearse en mayor proporción con machos silvestres. Sin embargo, cuando comparamos la aceptación de las hembras silvestre por machos de ambas cepas, encontramos que los machos LFS fueron aceptados en una mayor proporción que los machos LFM $(\mathrm{t}=3.52 ; \mathrm{df}=16, \mathrm{P}<0.05)$.

Competencia sexual. La evaluación de los insectos en competencia en jaulas de laboratorio, registró un mayor número de apareamientos cuando se usaron hembras LFS (figura 2), sin embargo la cantidad de apareamientos logrados por machos LFM fue significativamente menor que la cantidad alcanzada por machos LFS, tanto con hembras CFS ( $\mathrm{t}=8.76, \mathrm{df}=8, \mathrm{P}<0.05)$, como con hembras LFS $(\mathrm{t}=34.88, \mathrm{df}=8, \mathrm{P}<0.05)$. En la evaluación sin competencia los machos LFM y LFS no presentaron diferencias significativas $(\mathrm{t}=-0.60, \mathrm{df}=8, \mathrm{P}>0.05)$ en el número de apareamientos con hembras LFS.

Producción de feromonas. Los análisis de los volátiles emitidos durante los llamados sexuales (figura 3), reflejaron que no existen diferencias en los componentes feromonales entre los machos LFS y LFM. Además, tomando como 
referencia el estándar interno que fue inyectado a una concentración de $100 \mathrm{ng} / \mu 1$ en ambas muestras, no se observaron diferencias significativas en la cantidad emitida de volátiles $(Z-3$ nonenol $\mathrm{U}=46, \mathrm{P}>0.05 ; \mathrm{Z}, \mathrm{Z}-3,6$ nonadienol $\mathrm{U}=30, \mathrm{P}>0.05$; suspensolide $U=44, P>0.05$; $E, E-\cdot$ farneseno $Z=48, P>0.05$; anastrefina $U=46$, $\mathrm{P}>0.05$; epianastrefina $\mathrm{U}=41, \mathrm{P}>0.05$ ).

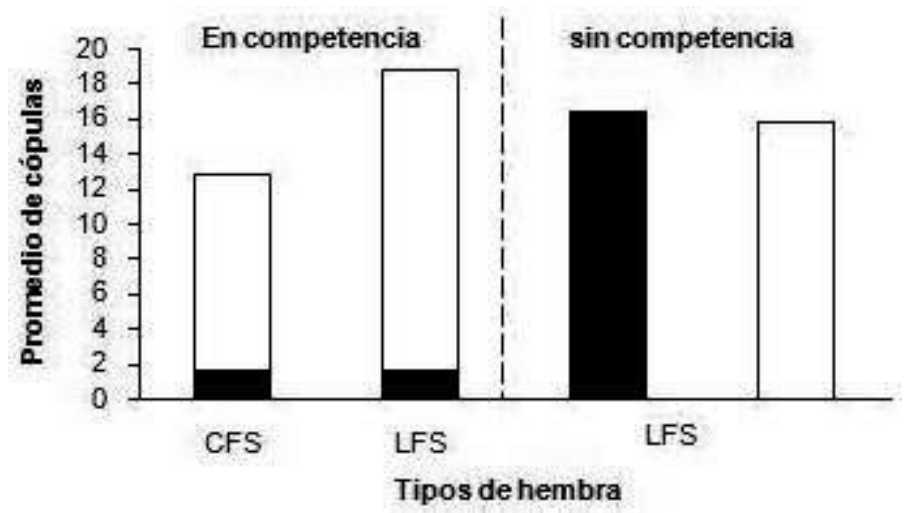

Figura 2. Promedio de apareamientos registrados en competencia y sin competencia de $A$. ludens, las barras blancas representan a machos LFS y las barras negras a los LFM.

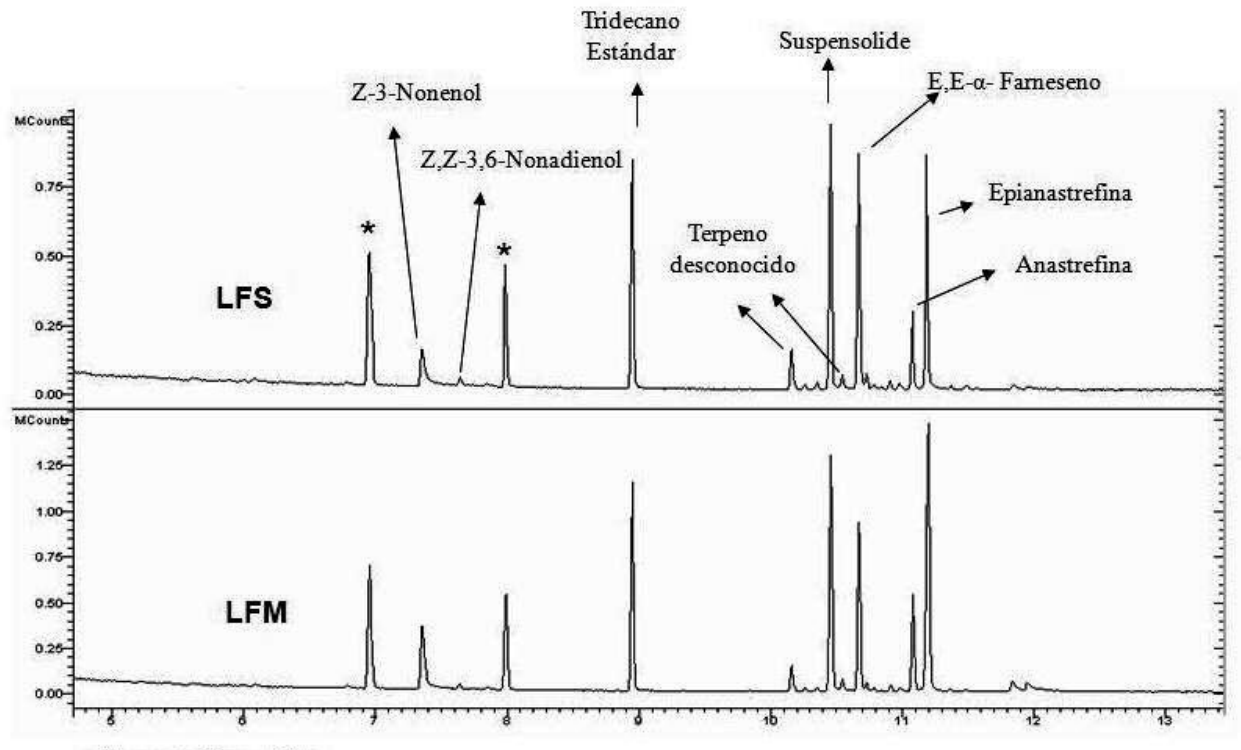

* Compuesto desconocido

Figura 3. Cromatogramas obtenidos por GC de volátiles de machos LFM y LFS de $A$. ludens emitidos durante el llamado sexual. 


\section{DISCUSIÓN}

Los datos de los atributos biológicos (fecundidad, fertilidad y supervivencia) para los estados inmaduros, indican que los insectos LFM poseen la misma capacidad que las moscas LFS y por lo tanto pueden ser criados bajo las mismas condiciones. Estas características han sido reportadas en especies como C. capitata (Rossler, 1979; Niyazi et al., 2005), A. fraterculus (Yamada \& Selivon, 2001) y recientemente en Bactrocera cucurbitae (McInnis et al. 2004, Harris et al. 2006) donde se estudió ampliamente la biología y el comportamiento sexual. Trabajos similares también fueron realizados por Saul y McCombs (1992) con mutantes de C. capitata (Wiedemann), B. dorsalis (Hendel) y B. cucurbitae (Coquillet), ellos concluyeron que las mutantes de ojos we, we y le, poseen una capacidad reproductiva similar a sus homólogos tipo silvestre y permiten una rápida identificación en campo, que los coloca como candidatas a ser usadas como marcadores genéticos en programas de control. Sin embargo, aunque ellos no evaluaron los insectos en competencia en campo, discuten las posibles desventajas que estos pudieran presentar en campo, como las encontradas en este estudio.

En nuestras evaluaciones, la comparación de las pruebas de compatibilidad sexual entre las cepas LFS y LFM con insectos silvestres, nos indicó de manera contundente que los insectos silvestres son significativamente menos compatibles con los insectos LFM que con los LFS. También, que las hembras silvestres claramente pudieron discriminar entre machos CFS, LFS y LFM, desviando su apareamiento significativamente en el orden mencionado. Este comportamiento es similar al reportado en las hembras silvestres de $C$. capitata, donde estas pueden discriminar entre machos de campo y de cría masiva (Shelly et al. 1994, Cayol et al. 1999, Lance et al. 2000). Mientras que las hembras LFS y LFM de manera similar, presentaron una frecuencia de apareamiento más alto con machos de laboratorio que con los de campo. Esta diferencia de comportamiento entre hembras de campo y laboratorio ha sido ampliamente descrita y varios autores sugieren que las hembras de laboratorio pierden capacidad de elección de pareja después de varias generaciones en condiciones artificiales y bajo presiones poblacionales como las implicadas en la cría masiva (Kaneshiro 1993, Iwahashi 1996, Liedo et al. 1996, Briceño et al. 2000).

En los machos, a pesar de que en la emisión de feromonas sexuales no se encontraron alteraciones tanto en calidad (componentes feromonales) como en cantidad, las pruebas de competencia evidenciaron que los machos LFM son deficientes para conseguir pareja cuando se encuentran en competencia, aun utilizando hembras de laboratorio con bajo poder selectivo. De tal manera, considerando las diferentes condiciones de comportamiento de las dos hembras usadas (cría y de campo), podemos deducir que el bajo desempeño de los machos mutados no fue debido al poder de selección de la hembra y si a la pérdida de ciertos atributos de cortejo originados de la colonización o mutación. Durante la 
colonización, a pesar de que la cepa mutada fue restaurada con insectos silvestres es probable que la colonización con pocos individuos haya ejercido una fuerte presión de selección artificial y en consecuencia los machos presentan un comportamiento anormal de cortejo que resulta en el rechazo de hembras silvestres (Rull \& BarredaLanda 2007). Tampoco descartamos que esta discriminación sea originada por la coloración de ojos. En algunos insectos se ha demostrado que la coloración de ojos sirve como medio de comunicación durante el cortejo (Burk 1981). De esta forma se menciona que en $C$. capitata la coloración de ojos y las setas orbitales producen las señales visuales masculinas (Eberhard 1994), así como en Phytalmia megalotis los colores faciales y de ojos contribuyen como advertencia en la exhibición del tamaño entre los machos rivales (Dodson 1997). Esto indica el papel de la visión en los insectos y la posible causa de discriminación de los insectos mutados con insectos silvestres.

En la TIE todos los aspectos relacionados al comportamiento sexual de los insectos son importantes, ya que de ello depende el éxito o fracaso de los programas. En conclusión, los resultados de este estudio indican que a pesar de que la mutante no difiere significativamente con los insectos de cría en sus atributos biológicos y en la emisión de feromona sexual (lo cual es indicativo de que puede ser adaptado a las condiciones de cría masiva), no se recomienda el uso directo de esta mutación como marcador genético para programas de liberación de la TIE, debido a que los machos presentan bajo desempeño sexual cuando se relacionan con hembras silvestres.

AGRADECIMIENTOS. Se agradece a Marco P. Pérez, José L. Zamora y Jesús A. Escobar (Moscafrut) por su invaluable asistencia técnica. También agradecemos a Refugio Hernández (Moscafrut) por el soporte técnico empleado durante la planificación y desarrollo del proyecto. Esta investigación fue financiada por la campaña nacional contra moscas de la fruta (CNCMF) DGSVSAGARPA.

\section{LITERATURA CITADA}

AIEA, USDA, FAO. 2003. A manual of Quality Control for fruit flies. Version 5.

Aluja, M., J. Hendrichs \& M. Cabrera. 1983. Behavior and interaction between Anastrepha ludens (L.) and Anastrepha obliqua (M) on field caged mango tree. I. Lekking behavior and male territorialy. Pp: 122-133. In: R. Cavalloro (Ed). fruit flies of economic importance. CEC/IBOC Simposium. Athens, Grecce.

Briceño, D. Ramos \& W. G. Eberhard. 2000. Aggresive Behavior in Medflies (Ceratitis capitata) and its Modification by Mass Rearing (Diptera: Tephritidae). Journal of the Kansas Entomological Society. 72: 17-27.

Burk, T. 1981. Signaling and sex in acalyptrate flies. Florida Entomologist. 64: 30-43.

Cayol, J. P., J. Vilardi, E. Rial \& M. T. Vera. 1999. New indices and method to measure the sexual compatibility and mating performance of medfly laboratory reared strains under field cage conditions. Journal of Economic Entomology. 92: 140-145.

Coppedge, J. R., J. P. Spencer, H. E. Brown, C. J. Whitten \& J. W. Snow. 1979. A new dye marking technique for screwworm. Journal of Economic Entomology. 25:29-38. 
Dodson, G. N. 1997. Resourse defense mating system in antlered flies, Phytalmia spp. (Diptera: Tephritidae). Annals of the Entomological Society of America. 90: 496-504.

Eberhard, W. G. 1994. Evidence for widespread courtship during copulation in 131 species of insects and spiders, and implications for cryptic female choice. Evolution. 48: 711-733.

Enkerlin, W. L. López \& H. Celedonio. 1996. Increased accuracy in discrimination between captured wild unmarked and released dye-marked adults in fruit fly (Diptera: Tephritidae) sterile released programs. Journal of Economic Entomology. 89: 946-949.

Enkerlin, W. R. 2005. Impact of fruit fly programmes using the sterile insect technique. Pp. 651-676. In: V. A. Dyck, J. Hendrichs, and A. S. Robinson (Eds.). Sterile insect technique. Principles and practice in area-wide integrated pest management. International Atomic Energy Agency. Springer, Amsterdam, The Netherlands.

Hangler, J. R. \& C. G. Jackson. 2001. Methods for marking insects: current techniques and future prospects. Annual Review of Entomology. 46: 511-543.

Harris, E. J., T. E. Mangine \& G. K. Uchida. 2006. Field discovery of a pearly eye melon fly, Bactrocera cucurbitae (Hendel) (Diptera: Tephritidae), mutant. Proceedings of the Hawaiian Entomological Society. 38: 123-126.

Hernández-Ortíz, V. \& M. Aluja. 1993. Listado de especies del género neotropical Anastrepha (Diptera: Tephritidae) con notas sobre su distribución y plantas hospederas. Folia Entomológica Mexicana. 88: 89-105.

Hernández, E., T. Artiaga \& J. Domínguez. 2005. Métodos para la colonización y cría de moscas de la fruta. Pp. 21: 155-170. In: Memorias del XVI curso internacional sobre moscas de la fruta. Metapa de Domínguez, Chiapas, México.

Iwahashi, O. 1996. Problems encountered during long-term SIT program in Japan. Pp. 391-398. In: B. A. Mcpheron and G. J. Steck (Eds). Fruit Fly Pests. A word assessment of their biology and management. St. Lucie Press, Delray Beach, Florida.

Kaneshiro, K. Y. 1993. Introduction, colonization, and establishment of exotic insect populations: Fruit flies in Hawaii and California. American Entomologist. 39: 23-29.

Khoury, N. \& C. Abi. 2001. Influence of some holding/packaging factors and procedures on medfly pupae quality during storage. Research Co-ordination meeting, Mendoza Argentina, November.

Knipling, E. F. 1953. Possibilities of insect control or eradication through the use of sexually sterile males. Journal of Economic Entomology. 48: 456-462.

Liedo, P., J. Toledo \& M. I. Barrios. 1996. Comparación de los parámetros demográficos de una cepa silvestre y una de laboratorio de Anastrepha ludens (Díptera: Tephritidae). Pp. 33-34. In: Proceedings of $2^{\text {th }}$ Meeting of the Working Group on Fruit Flies of the Western Hemisphere. Viña del Mar, Chile.

Lance, D. R., D. O. McInnis, P. Rendon \& C. G. Jackson. 2000. Courtship among sterile and wild Ceratitis capitata (Diptera: Tephritidae) in field cages in Hawaiii and Guatemala. Annals of the Entomological Society of America. 93: 1179-1185.

McInnis, D. O., D. R. Lance \& C. G. Jackson. 1996. Behavioral resistance to the sterile insect release technique by the Mediterranean fruit fly (Diptera: Tephtritidae) in Hawaii. Annals of the Entomological Society of America. 89: 739-744.

McInnis D.O., S. Tan, R. Lim, J. Komatsu \& C. Albrecht. 2004. Development of a pupal color Based Genetic Sexing Strain of the Melon Fly, Bactrocera cucurbitae (Coquillett) (Diptera:Tephritidae). Annals of the Entomological Society of America. 97: 1026-1033.

Meza, J. S., E. Hernández, M. Salvador-Figueroa \& L. Cruz- López. 2002. Sexual compatibility, mating performance and sex pheromone release of mass-reared and wild Anastrepha obliqua (Diptera: Tephritidae) under field-cage conditions. Pp. 99-104. In: Proceeeding of $6^{\text {th }}$ international fruit fly symposium. Stellenbosch, South Africa. 
Meza, J. S., C. S. Zepeda, J. C. Domínguez \& D. Orozco. 2004. New mutant in the Mexican Fruit fly (Anastrepha ludens) and their possible role in the genetic sexing. P. 91. In: $5^{\text {th }}$ meeting of the Working Group on Fruit Flies of the Western Hemisphere. Florida, USA.

Meza-Hernández, J. S. \& F. Díaz-Fleisher. 2006. Comparison of Sexual Compatibility Between Laboratory and Wild Mexican Fruit Flies Under Laboratory and Field Conditions. Journal of Economic Entomology. 99: 1979-1986.

Niyazi, N., C. Caceres, A. Delprat, V. Wornoayporn, E. Ramírez, G. Franz \& A. S. Robinson. 2005. Genetics and Mating Competitiveness of Ceratitis capitata (Diptera: Tephritidae) Strains Carrying the Marker Sergeant, Sr2. Annals of the Entomological Society of America. 98: 119-125.

Norrbom, A. L. \& K. C. Kim. 1988. A list of the reported host plants of the species of Anastrepha (Diptera: Tephritidae). United States Department of Agriculture. (APHIS-PPQ). No. 81-52. P.114

Norrbom, A. L., R. A. Zucchi \& V. Hernández-Ortíz. 2000. Phylogeny of the genera Anastrepha and Toxotrypana (Trypetinae: Toxotrypanini) based on morphology. Pp. 299-342. In: M. Aluja and A. Norrbom (Eds). Fruit flies (Tephritidae): phylogeny and evolution of behavior. CRC, Press, Boca Raton, FI.

Norris, K. R. 1957. A method of marking Calliphoridae (Diptera) during emergence from the puparium. Nature. 180: 1002.

Parker, A. \& K. Mehta. 2007. Sterile insect technique: A model for dose optimization for improved sterile insect quality. Florida entomologist. 90: 88-95.

Reyes, J., G. Santiago \& P. Hernández. 2000. Mexican fruit fly eradication programme. Pp. 377-380. In: K. H. Tan (ed.). Area-wide control of fruit flies and other insect pests. Penerbit Universiti Sains Malaysia, Pulau Pinang, Malaysia.

Rössler, Y. 1979. The genetic of the Mediterranean fruit fly a white pupae mutant. Annals of the Entomological Society of America. 72: 583-585.

Rull, J. \& A. Barreda-Landa. 2007. Colonization of Hybrid to restore male Anastrepha ludens (Diptera: Tephritidae) mating competitiveness for sterile insect technique programs. Journal of Economic Entomology. 100: 752-758.

Saul, S. H. \& Mc. S. D. Combs. 1992. Light eye color mutants as genetic markers for released populations of Hawaiian fruit flies (Diptera: Tephritidae). Journal of Economic Entomology. 85:12401245 .

Schroeder, W. J. \& W. C. Mitchell. 1981. Marking Tephritidae fruit fly adults in Hawaii for releaserecovery studies. Proceedings of the Hawaiian Entomological Society. 23: 437-440.

Shelly, T. E., T. S. Whittier \& K. Y. Kaneshiro. 1994. Sterile insect release and the natural mating system of the Mediterranean fruit fly, Ceratitis capitata (Diptera: Tephritidae). Annals of the Entomological Society of America. 89: 754-758.

Yamada, M. S. \& D. Selivon. 2001. Rose, an Eye Color Mutation in a Species of the Anastrepha fraterculus Complex (Diptera: Tephritidae). Annals of the Entomological Society of America. 94: 592595.

Zar, J. H. 1999. Biostatistical analysis, $4^{\text {th }}$ ed. Prentice Hall, Englewood Cliffs, New Jersey, USA.

Recibido: 26 de febrero de 2008

Aceptado: 1 de septiembre de 2008 